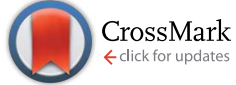

Cite this: RSC Adv., 2016, 6, 70943
Received 16th May 2016

Accepted 20th July 2016

DOI: $10.1039 / c 6 r a 12650 b$

www.rsc.org/advances

\section{Enhanced magnetic properties in ordered oriented ferrofibres $\uparrow$}

\begin{abstract}
Komal Jain, ${ }^{\text {ab }}$ Saurabh Pathak ${ }^{\mathrm{ab}}$ and R. P. Pant ${ }^{\star a b}$
We report the fabrication of oriented nano-ferrofibres using a typical electrospinning process in the absence of an applied magnetic field to assist alignment. For this, a double surfacted magnetic fluid was used to optimise and enhance the charging and thereby alignment of the fibres. The induced electric field in the PVA solution assists the orientation of magnetic nanoparticles inside the fibres. The microwave resonance studies show that the ferrofibres obtained have high magnetisation values $(1.2-3.7$ emu $\mathrm{g}^{-1}$ ) and uniaxial anisotropy with high effective anisotropy field values.
\end{abstract}

\section{Introduction}

Nano-ferrofibres are an intense area of contemporary research owing to their unique magneto-elastic and magneto-restrictive properties and applications such as EMI shielding, magnetic filters, sensors, low frequency magnetic shielding and magnetic induction..$^{1-5}$ However, for fibres to be used in an application they need to have high magnetisation and a preferred orientation. The size-shape distribution, magnetic properties, and surfactant coating (if any) of magnetic fillers in ferrofibres play vital roles in determining the nature of these fibres and their alignment during fabrication. There are various methods available for the preparation of ferrofibre such as melt, solution and gel state spinning. ${ }^{6,7}$ All these techniques use mechanical forces to create elongated filaments which on solidification form fibres which limits the fiber diameter.

Electrospinning uses electric forces to produce polymer jets by overcoming viscous force and surface tension to form microfibres to nanofibres. The diameter and length of fibre can be tuned by controlling viscoelasticity of precursors, spinning time and potential difference etc. ${ }^{8,9}$ Due to the bending instability of polymer jet during electrospinning, the formation of aligned fibres with uniform diameter remains a challenge. Researchers have developed various methods such as using metal substrate, sharp needles, copper wire frame collector drum, magnetic electrospinning etc. to improve positioning and alignment of fibres. ${ }^{\mathbf{1 0 - 1 4}}$ In this, the production of smooth textured ferrofibers is hampered by beading in fibres due to agglomeration of magnetic particles.

\footnotetext{
${ }^{a}$ Acadamey of Scientific and Innovative Research, CSIR-NPL Campus, New Delhi, India ${ }^{b}$ EPR \& Magnetic Fluid Division, CSIR-National Physical Laboratory, New Delhi 110012, India.E-mail: rppant@nplindia.org

† Electronic supplementary information (ESI) available. See DOI: 10.1039/c6ra12650b
}

In order to overcome the beading problem, we have used double surfacted water based $\mathrm{Fe}_{3} \mathrm{O}_{4}$ magnetic fluid (saturation magnetisation $150 \mathrm{G}$ ) with particle size in the range of 8-13 nm of spherical shape to improve homogeneity and orientation of the particles in ferrofibres. To obtain uniformity and homogenise magnetic nanoparticles/polymer solution was maintained by ultrasonication before electrospinning.

\section{Experimental}

Water based magnetic fluid (FFW) was prepared using chemical coprecipitation method. ${ }^{15} \mathrm{Fe}_{3} \mathrm{O}_{4}$ nanoparticles were coprecipitated using $\mathrm{FeCl}_{3}$ and $\mathrm{FeCl}_{2}$ (Merck chemicals) in $2: 1$ molar ratio with oleic acid as primary surfactant and 35\% ammonia solution (Merck chemicals) as precipitating agent. The $\mathrm{pH}$ of solution was maintained at 10 and temperature $80{ }^{\circ} \mathrm{C}$ during reaction under constant stirring. The precipitate was washed with deionised water till $\mathrm{pH}$ is neutralised. Thus, obtained $\mathrm{Fe}_{3} \mathrm{O}_{4}$ particles were dispersed in sodium oleate solution where sodium oleate acts as secondary surfactant to get FFW.

Thereafter, three samples ' $a$ ', ' $b$ ' and 'c' of ferrogel were prepared by mixing FFW with $5 \%, 10 \%$ and $15 \% \mathrm{w} / \mathrm{w}$ in bubble free $10 \% \mathrm{w} / \mathrm{w}$ PVA solution. The solution was homogenised by subjecting to continuous stirring for 14 hours at $120 \mathrm{rpm}$ at room temperature. Thus a uniform dissolution of magnetic fluid in polymer solution was obtained. A series of experiments were performed to optimise the visco-elastic properties of ferrogel samples using magneto-rheometer (Anton Paar MCR 301). The ferrogel samples with viscosity in the range of 650-700 $\mathrm{cP}$ were used for electrospinning process.

A typical electrospinning setup has been used where collector drum is grounded as shown in Fig. 1(a). Ferrogel was pumped through a stainless steel needle at a rate of $0.2 \mathrm{ml}$ per hour using a syringe pump. A potential difference of $20 \mathrm{kV}$ was maintained between the needle and collector drum. The collector drum was kept at a distance of $20 \mathrm{~cm}$ from the syringe 


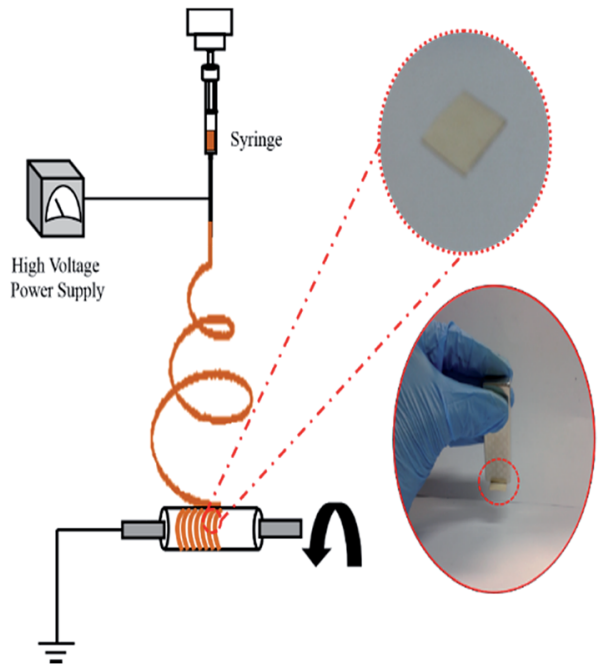

(a)

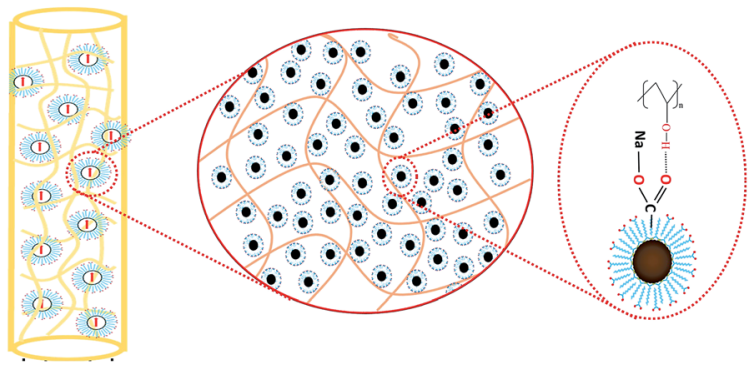

(b)

Fig. 1 (a) Schematic of electrospin set up for making ferrofiber (b) oriented double surfacted $\mathrm{Fe}_{3} \mathrm{O}_{4}$ nanoparticles in PVA matrix.

and rotated at a speed of $2000 \mathrm{rpm}$. Thus a sheet of ferrofibres with uniform distribution of magnetic particles was obtained after 10 hours of electrospinning process.

\section{Results and discussion}

Fig. 2 show the optical microscope images of ferrofibres of sample a and b after 5 min of electrospinning. The images show the increased orientation of fibres with the increase in FFW concentration with sample a (Fig. 2(a)) showing complete random deposition of fibres as compared to the sample b (Fig. 2(b)). FE-SEM micrographs of ferrofibres (Fig. 2(c-e)) recorded after a deposition of ferrofibres for 10 hours show nearly uniform diameter $(\sim 100-200 \mathrm{~nm})$ and smooth texture embedded with surfactant coated $\mathrm{Fe}_{3} \mathrm{O}_{4}$ nanoparticles. An increase in fibres diameter with the increase in magnetic fluid concentration is observed. Further, the ordered alignment in a preferred direction enhances with increment in magnetic fluid weight percentage is due to increased ion dipole interaction. Fig. 2(e) show well-ordered alignment of ferrofibres in sample ' $c$ ' along the axis of fiber formation with some entanglement. Whereas a complete entanglement of ferrofibres formation is observed in sample 'a' Fig. 2(c). The alignment of ferrofibres with increase in FFW concentration in precursor ferrogel is believed to be due increase in carboxylic group which enhances the charging
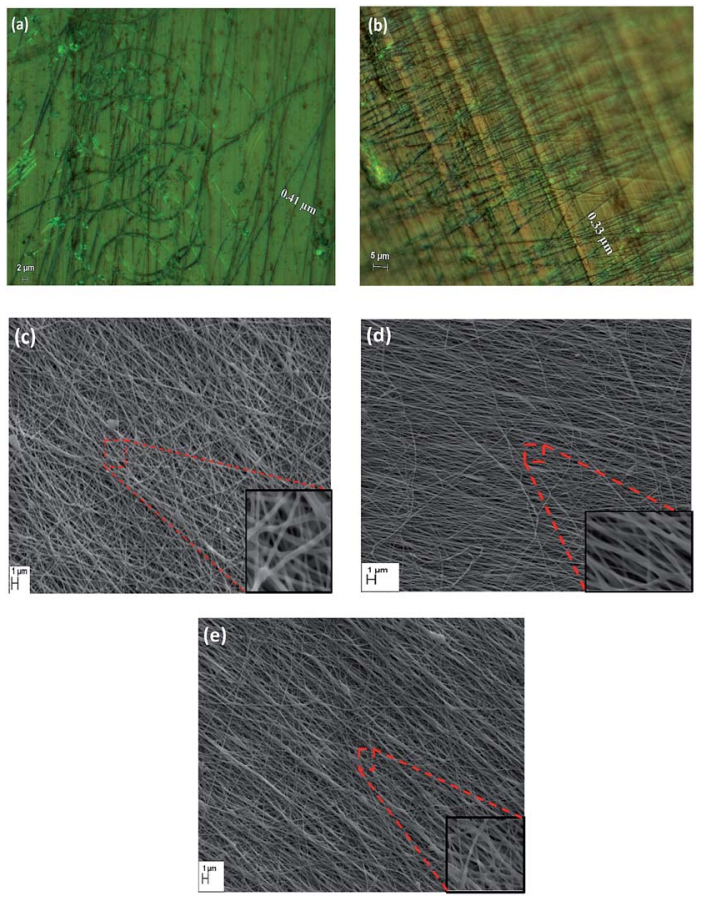

Fig. 2 ( $a$ and $b)$ Optical micrographs of ferrofibres with $5 \%$ and $10 \% \mathrm{w} /$ W FFW in precursor ferrogel. (c-e) FE-SEM images show the aligned ferrofiber SEM micrographs of sample a, b, c.

of ferrogel. The enhanced charge results in better alignment of the fibres. Here, the double surfactant in FFW serves three purpose of (1) particle growth inhibitor during magnetic fluid synthesis, (2) stabilising agent and (3) binder between magnetic nanoparticles and carrier water and also acting as binding agent between nanoparticles and PVA chain. Yet another, sodium oleate as secondary surfactant enhances the adsorption of PVA on the surface of $\mathrm{Fe}_{3} \mathrm{O}_{4}$ nanoparticles and also induces a negative charge on the surface. ${ }^{16}$ Thereby, enhancing the charging process in ferrofibres further which get deposited on the collector well separated due to electrostatic repulsion and gets aligned parallel to each other. The hydrogen bonding between -OH of PVA, carboxylic $(-\mathrm{COOH})$ group of surfactant and water molecules form a homogenised structure of ferrogel system, which after electrospun gives ferrofibres with oriented $\mathrm{Fe}_{3} \mathrm{O}_{4}$ nanoparticles bound between PVA polymer chains Fig. 1(b). Further, oleic acid acting as primary surfactant gets covalently bonded to the $\mathrm{Fe}_{3} \mathrm{O}_{4}$ nanoparticles reducing the surface spin disorder causing alignment in a preferred direction and enhanced magnetisation. ${ }^{17}$

The XRD measurement were performed using Rigaku XRG 2 kW X-ray diffractometer. Fig. 3(a) show X-ray diffraction pattern of the ferrofiber composites. The broad diffraction peak at $19.5^{\circ}$ corresponds to PVA, whereas small peaks at $35.7^{\circ}, 57.1^{\circ}$ and $62.5^{\circ}$ $2 \theta$ value belong to (311), (511) and (440) reflections of $\mathrm{Fe}_{3} \mathrm{O}_{4}$ nanoparticles crystal planes. The structural parameters of $\mathrm{Fe}_{3} \mathrm{O}_{4}$ nanoparticles embedded in polymer matrix were determined using Williamson-Hall method $\beta \cos \theta=4 \varepsilon \sin \theta+\frac{\lambda}{D}$ giving values $9.4(1) \mathrm{nm}$ and 0.0006 for crystallite size $(D)$ and strain $(\varepsilon)$ respectively (inset Fig. 3(a)). Fig. 3(b) show the $M-H$ loop showing 


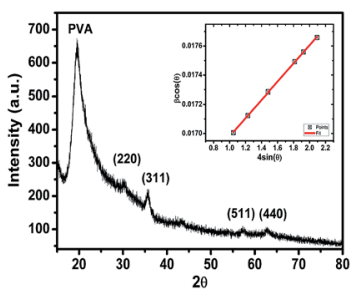

(a)

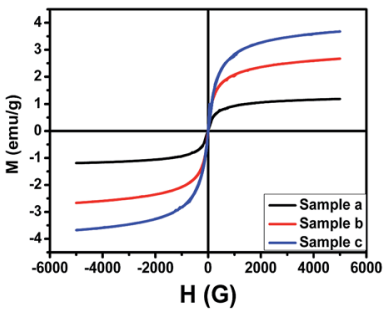

(b)
Fig. 3 (a) XRD pattern of ferrofiber, inset shows the Williamson-Hall plot of $\mathrm{Fe}_{3} \mathrm{O}_{4}$ nanoparticles (b) $\mathrm{M}-\mathrm{H}$ loop show superparamagnetic nature of ferrofibres.

the superparamagnetic nature of ferrofibres. Magnetic parameters are given in Table 1 . The FFW particles show no coercivity and retentivity due to their superparamagnetic nature. However, ferrofibres show some residual magnetic field due to the conjunction of particles in polymer matrix as shown in Fig. 4.

The anisotropic effect of aligned ferrofibres was studied by microwave absorption with angular variation using ferromagnetic resonance (FMR, Bruker Biospin A300) in X-band (9.54 $\mathrm{GHz}$ ). Here sample ' $\mathrm{b}$ ' and sample 'c' are chosen because these samples show preferred orientation of ferrofibres as observed in electron micrographs. Fig. 5(a) show the microwave resonance spectra of in plane $(\|)$ and out of plane $(\perp)$ for both the sample at $300 \mathrm{~K}$. A broad resonance signal indicates the ferromagnetic nature of ferrofibres. A shift in resonance field $\left(H_{\text {res }}\right)$ with angle confirms the orientation of magnetic domains in ferrofibres. Further, in-plane configuration a short relaxation behaviour at $H_{\mathrm{r}}$ $=\sim 3380 \mathrm{G}$ (inset Fig. 5(a)) confirm the superparamagnetic nature of $\mathrm{Fe}_{3} \mathrm{O}_{4}$ nanoparticles found in magnetic fluid. ${ }^{15,18}$ However, the absence of same signal in out of plane $(\perp)$ geometry confirms the existence of magnetic anisotropy due to alignment of the domains in ferrofibres. In out of plane configuration, the relaxation of superparamagnetic particles is strongly coupled with fibres and occur via Brownian relaxation dominantly

Table 1 Magnetic parameters value

\begin{tabular}{llll}
\hline \multicolumn{5}{c}{ Physical quantity } & \\
\cline { 2 - 4 } Sample & $\begin{array}{l}\text { Saturation magnetisation } M_{\mathrm{S}} \\
\left(\mathrm{emu} \mathrm{g}^{-1}\right)\end{array}$ & $\begin{array}{l}\text { Coercivity } \\
(\mathrm{G})\end{array}$ & $\begin{array}{l}\text { Retentivity } \\
(\mathrm{G})\end{array}$ \\
\hline a & 1.18 & 6.14 & 1.8 \\
b & 2.68 & 7.4 & 7.2 \\
c & 3.66 & 8.98 & 8.4
\end{tabular}
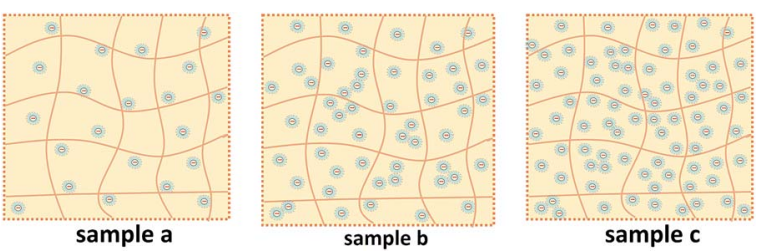

Fig. 4 Schematic showing aggregation in ferrofibres. (a)

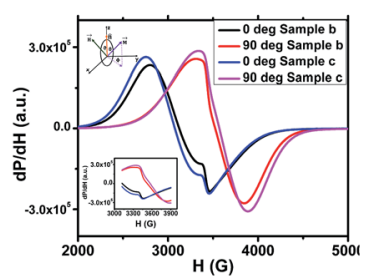

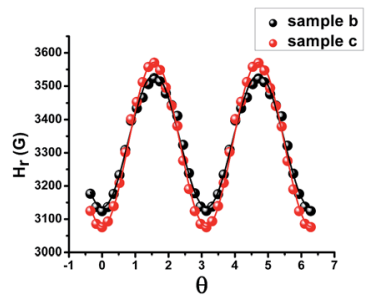

(b)
Fig. 5 (a) FMR spectra at $0^{\circ}$ and $90^{\circ}$, inset show the appearance of shoulder at $3380 \mathrm{G}$ due to superparamagnetic nature of $\mathrm{Fe}_{3} \mathrm{O}_{4}$ nanoparticles. (b) show the angle dependent microwave resonance at room temperature showing uniaxial anisotropy.

resulting in merging of small peak in the broad signal. Whereas in-plane geometry the relaxation mechanism is mainly via short Neel relaxation showing superparamagnetic signal of $\mathrm{Fe}_{3} \mathrm{O}_{4}$ nanoparticles embedded in the polymer matrix. The angular dependence of resonance field $\left(H_{\text {res }}\right)$ for both samples ' $b$ ' and ' $c$ ' is fit using simple equation (eqn (1)) as shown in Fig. 5(b).

$$
\frac{H_{\text {res }}(\theta)-H_{\text {res }}\left(0^{\circ}\right)}{H_{\text {res }}\left(90^{\circ}\right)-H_{\text {res }}\left(0^{\circ}\right)}=\sin ^{2}(\theta)
$$

According to magnetic resonance theory, the resonance condition for ferrimagnetic and ferromagnetic particles with uniaxial anisotropy ${ }^{\mathbf{1 5 , 1 8}}$ is given by

$$
H_{\text {res }}=\frac{\omega}{\gamma}-H_{\mathrm{a}} P_{2} \cos (\theta)
$$

where $\gamma$ is gyromagnetic ratio, $\omega$ is angular frequency of microwave, $H_{\mathrm{a}}$ anisotropy field, $P_{2}(\cos \theta)$ is the second-order Legendre polynomial, and $\theta$ is the angle between the axis of the particle and field. According to eqn (2) for a completely aligned system, $H_{\text {res }}$ for parallel $\left(\theta=0^{\circ}\right)$ and perpendicular $(\theta=$ $90^{\circ}$ ) configurations is given by

$$
\begin{aligned}
H_{\text {res }}\left(0^{\circ}\right) & =\frac{\omega}{\gamma}-H_{\mathrm{a}} \\
H_{\text {res }}\left(90^{\circ}\right) & =\frac{\omega}{\gamma}+\frac{1}{2} H_{\mathrm{a}}
\end{aligned}
$$

For magnetic fluid, there exist no preferred orientation direction at room temperature and resulting in zero anisotropy in the magnetic fluid samples. But FMR results of ferrofiber confirm the preferential orientation giving rise to uniaxial anisotropy field. The fitted observed data under Morais model ${ }^{15,19}$ gives an insight to the intrinsic magnetic anisotropy behaviour of ferrogel fibres. Using the value of $I$ obtained from Langevin function fit on VSM data (Table 1S: ESI $\dagger$ ), the theoretical fit can be performed using eqn (5) to obtain value of uniaxial magnetic anisotropy energy constants.

$$
H_{\mathrm{res}}=H_{\mathrm{EF}}-H_{\mathrm{X}}-H_{\mathrm{EK}}
$$

where $H_{\mathrm{EK}}=\frac{K_{\mathrm{EK}}}{I}\left(3 \cos ^{2} \theta-1\right)$ is effective uniaxial anisotropy field, $H_{\mathrm{EF}}$ : effective magnetic field, $H_{\mathrm{X}}$ : exchange anisotropy 
Table 2 Fitted FMR parameters

\begin{tabular}{llll}
\hline & \multicolumn{3}{l}{ Fitted parameters } \\
\cline { 2 - 4 } Sample & $H_{\mathrm{a}}(\mathrm{G})$ & {$\left[\frac{K_{\mathrm{EK}}}{I}\right](\mathrm{G})$} & $\begin{array}{l}H_{\mathrm{EF}}- \\
H_{\mathrm{X}}(\mathrm{G})\end{array}$ \\
\hline $\mathrm{b}$ & 266.4 & 132.9 & 3400 \\
$\mathrm{c}$ & 329.6 & 164.7 & 3407 \\
\hline
\end{tabular}

field, I: magnetisation of nanoparticle and $K_{\mathrm{EK}}$ : effective anisotropy constant.

From Table 2, the quantitative effect of increasing magnetic filler content can be seen is clearly visible in values. This can be correlated with observed thickness of fibres in FE-SEM images. The increase in $H_{\mathrm{a}}$ and $K_{\mathrm{EK}} / I$ values is considerable. However, the difference in $\left[H_{\mathrm{EF}}-H_{\mathrm{X}}\right]$ values is not substantial.

\section{Conclusion}

In summary, the present work reports a novel method to prepare aligned magnetic nanofibres using electrospin technique without any application of external magnetic field. This method can be used for any type of magnetic fluid composition with double surfactant coating in polymer matrix used to prepare desired ferrofibres. A control on the magnetic fluid concentration can greatly enhance the order of alignment due to surfactant molecules and diameter size for various application purposes. The magnetic measurements indicate that ferrofibres possesses high magnetisation and low coercivity (6-8 G) showing superparamagnetic behaviour. Further, microwave resonance investigations shows that these fibres have high uniaxial anisotropy values revealing the nature of interaction between superparamagnetic particles in polymer matrix. This type of fibres can be used for EMI shielding, air filters, sensors, magnetic induction etc.

\section{Acknowledgements}

Authors are thankful to Dr R. K. Kotnala for carrying out VSM measurements and Director, CSIR-National Physical Laboratory for his encouragement to carry out this work. Authors would like to thank Department of Science and Technology (DST) for funding the work under the project GAP123532.

\section{References}

1 J. S. Andrew, J. J. Mack and D. R. Clarke, J. Mater. Res., 2008, 23, 105-114.

2 J. S. Andrew and D. R. Clarke, Langmuir, 2008, 24, 8435-8438.

3 M. Wang, H. Singh, T. Hatton and G. Rutledge, Polymer, 2004, 45, 5505-5514.

4 D. A. Allwood, G. Xiong, C. Faulkner, D. Atkinson, D. Petit and R. Cowburn, Science, 2005, 309, 1688-1692.

5 R. Han, W. Li, W. Pan, M. Zhu, D. Zhou and F.-s. Li, Sci. Rep., 2014, 4, 7493.

6 M. E. Walter, US Pat., US 3179614A, 1965.

7 R. S. Irwin, US Pat., US 4640972A, 1987.

8 R. Kessick, J. Fenn and G. Tepper, Polymer, 2004, 45, 29812984.

9 M. Cloupeau and B. Prunet-Foch, J. Electrost., 1989, 22, 135159.

10 R. Dersch, T. Liu, A. Schaper, A. Greiner and J. Wendorff, J. Polym. Sci., Part A: Polym. Chem., 2003, 41, 545-553.

11 E. Zussman, A. Theron and A. Yarin, Appl. Phys. Lett., 2003, 82, 973-975.

12 P. Katta, M. Alessandro, R. Ramsier and G. Chase, Nano Lett., 2004, 4, 2215-2218.

13 G. Xu, S. Zhang, Q. Zhang, L. Gong, H. Dai and Y. Lin, Sens. Actuators, B, 2016, 222, 707-713.

14 H. Wang, H. Tang, J. He and Q. Wang, Mater. Res. Bull., 2009, 44, 1676-1680.

15 A. Shankar, M. Chand, G. A. Basheed, S. Thakur and R. P. Pant, J. Magn. Magn. Mater., 2015, 374, 696-702.

16 N. Labidi and A. Djebaili, J. Miner. Mater. Charact. Eng., 2008, 7, 147.

17 P. Guardia, B. Batlle-Brugal, A. Roca, O. Iglesias, M. Morales, C. Serna, A. Labarta and X. Batlle, J. Magn. Magn. Mater., 2007, 316, e756-e759.

18 F. Gazeau, J. Bacri, F. Gendron, R. Perzynski, Y. L. Raikher, V. Stepanov and E. Dubois, J. Magn. Magn. Mater., 1998, 186, 175-187.

19 P. Morais, M. Lara and K. S. Neto, Philos. Mag. Lett., 1987, 55, 181-183. 


\section{University Library}

\section{- M M I N E R VA A gateway to Melbourne's research publications}

Minerva Access is the Institutional Repository of The University of Melbourne

Author/s:

Jain, K;Pathak, S;Pant, RP

Title:

Enhanced magnetic properties in ordered oriented ferrofibres

Date:

2016-07-26

Citation:

Jain, K., Pathak, S. \& Pant, R. P. (2016). Enhanced magnetic properties in ordered oriented ferrofibres. RSC Advances: an international journal to further the chemical sciences, 6 (75), pp.70943-70946. https://doi.org/10.1039/C6RA12650b.

Persistent Link:

http://hdl.handle.net/11343/290764 